\title{
Benefits of urethroplasty for managing urethral amyloidosis: A case report
}

\author{
XIAOLIN YAO, YUFANG YUAN, JIAJIE FANG and HAI JIANG \\ Department of Urology, The First Affiliated Hospital, School of Medicine, \\ Zhejiang University, Hangzhou, Zhejiang 310003, P.R. China
}

Received June 28, 2016; Accepted June 16, 2017

DOI: $10.3892 /$ etm.2017.4983

\begin{abstract}
Urethral amyloidosis is a rare condition in which eosinophilic amyloid proteins are deposited in the urethra. Only a small number of reports on urethral amyloidosis have been published. Increased interest has been associated with this disease due to its clinical similarities with urothelial carcinoma. A biopsy of the lesion and a histological examination are essential for the correct diagnosis. Conservative management has been suggested by various urologists as the optimal treatment approach for urethral amyloidosis; however, recurrence and urethral stricture are common, and typically further treatment is required. Urethroplasty has been used in a limited number of urethral amyloidosis cases, with beneficial short-term outcomes; however, long-term follow-up data are lacking. The present case report describes the cases of 2 patients with urethral amyloidosis who underwent urethroplasty without recurrence or progression for $>2$ years. These findings indicate that urethroplasty is beneficial for the long-term management of urethral amyloidosis.
\end{abstract}

\section{Introduction}

Amyloidosis is a group of diseases in which eosinophilic amyloid proteins are deposited in multiple tissues (1). The deposits are characterized by apple-green birefringence under a polarized light microscope following Congo red staining. Amyloidosis may be primary, secondary or hereditary, and the involved area may be systemic or localized (2). System immune disorder is the basis behind this process, even though its appearance is localized in certain cases. Localized amyloidosis may appear at various sites, including the urinary tract (3).

Urethral amyloidosis is a worldwide rare condition and has a lower incidence rate compared with amyloidosis in the

Correspondence to: Dr Hai Jiang, Department of Urology, The First Affiliated Hospital, School of Medicine, Zhejiang University, 79 Qingchun Road, Hangzhou, Zhejiang 310003, P.R. China

E-mail: 13958011420@126.com

Key words: amyloidosis, buccal mucosa, pedicle flap, urethroplasty prostate or seminal vesicles (4). In the limited number of cases reported, urethral amyloidosis has occurred more often in men. However, urologists have investigated the urethral form of amyloidosis in depth due to its similarities to the clinical appearance of urothelial carcinoma (5). Urethral amyloidosis demonstrates a benign but progressive clinical course and always leads to a urethral stricture (4). Conservative management, including urethrotomy and dilatation, has been suggested for the treatment of urethral amyloidosis; however, only a limited number of patients have been treated with urethroplasty (4). Analyses of the long-term outcomes of these management approaches are lacking. In the present study, the cases of 2 patients with urethral amyloidosis who underwent urethroplasty without recurrence or progression for 2 years were reported. The findings of the present study indicate that urethroplasty is an appropriate option for the long-term management of urethral amyloidosis.

\section{Case report}

Between January and December 2013, a total of 2 male patients (39 and 59 years old) with recurrent hematuria and bloody secretions, but no history of urothelial carcinoma or sexually transmitted diseases, presented to the Department of Urology of the First Affiliated Hospital of Zhejiang University (Hangzhou, China) for antibiotic therapy. Informed written consent was obtained from each patient prior to the study. Furthermore, the present study was approved by the Ethics Committee of the First Affiliated Hospital of Zhejiang University.

Urethroscopy was used to evaluate the urethra, which revealed an anterior urethral neoplasm mimicking a malignant lesion (Fig. 1). Biopsies were performed by urethroscopy. The samples extracted from the body, fixed in $10 \%$ neutral formalin for $24 \mathrm{~h}$ at room temperature, paraffin-embedded, and the specimens were stained with hematoxylin and eosin stain (at room temperature for $15 \mathrm{~min}$ ) and pathological examination was performed using a light microscope (magnification, $\mathrm{x} 400$ ). Urothelial carcinoma was excluded as there was no evidence of malignancy; however, amyloidosis was diagnosed due to amyloid protein deposition and infiltration of plasmacytes (Fig. 2A). Amyloidosis was only indicated in the urethra by pathological examination without any evidence of systemic lesions. Urethroscopic results indicated that localized lesions were identified distally in the penile urethra, and 


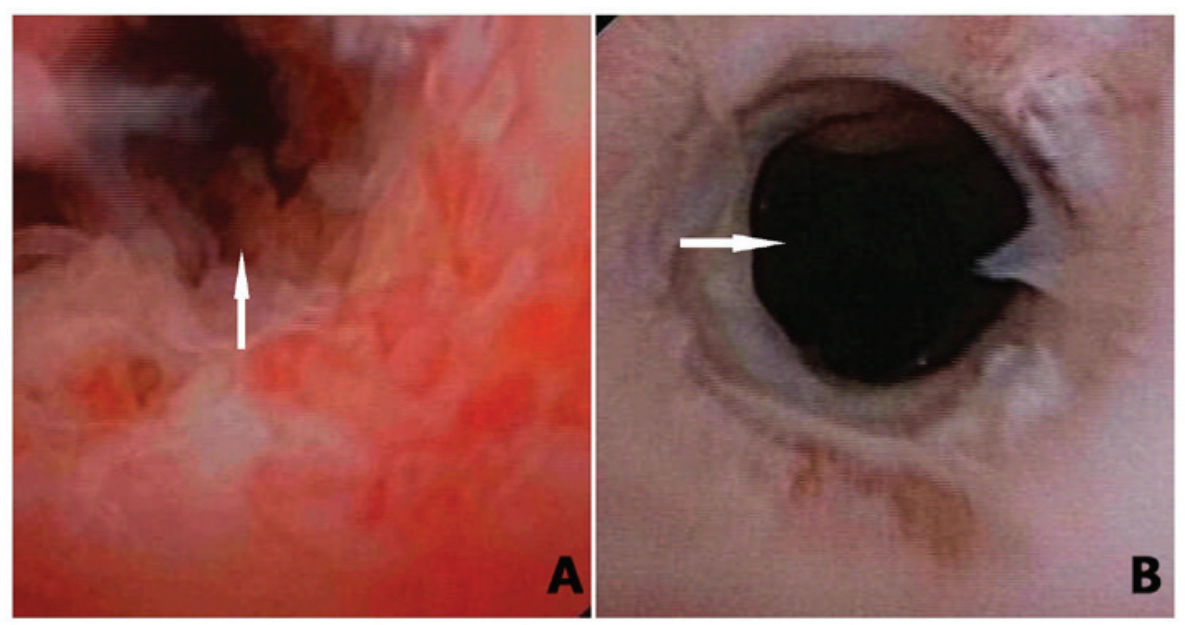

Figure 1. Urethroscopic findings (A) prior to and (B) post-urethroplasty. (A) Anterior urethral amyloidosis was indicated, which mimicked a malignant lesion (white arrow). (B) A smooth artificial urethra without stricture (white arrow).
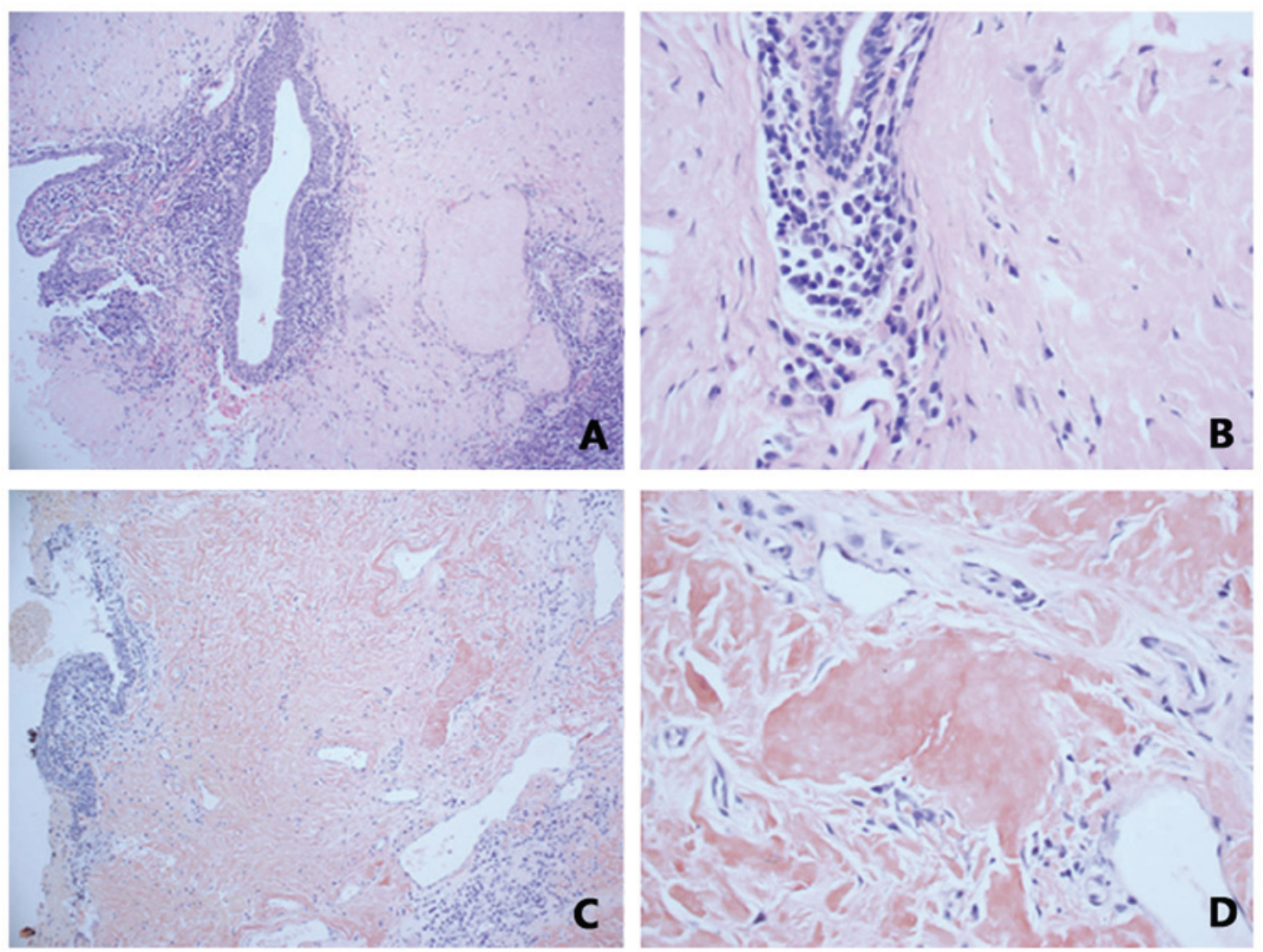

Figure 2. Microscopic findings of urethral amyloidosis. Hematoxylin and eosin staining revealed eosinophilic amorphous deposits in the sub-urothelial lamina propria at magnifications of (A) x100 and (B) x400. Amorphous indications were observed with Congo red staining at magnifications of (C) x100 and (D) x400.

$\sim 1$ and $2 \mathrm{~cm}$ proximal to the fossa navicularis. The urethras were affected in 4- and 7-cm segments, and extensive circular ulcerations and polypoid changes in the mucosa were detected (Fig. 1A). No end-to-end anastomosis was available following the complete resection of the lesions deep into the tunica albuginea on the dorsal side of the urethra, thus urethroplasty was the only treatment option. A pedicled penile skin flap was provided in 1 case, and a pedicled penile skin flap and buccal mucosa was used in the other case, which were applied according to the length of the urethral defect. An 8.0-cm-long and $1.5-\mathrm{cm}$-wide buccal mucosa graft was harvested from the patient with the longer lesion, which was modified and fixed on the tunica albuginea as the dorsal side of the urethra (data not shown). A pedicled penile skin flap of the same size was used for the ventral side. These 2 grafts were anastomosed with the native urethra in order to rebuild a new urethra. A 4.0-cm-long and 2.5-cm-wide pedicled penile skin flap was used for the other patient with the shorter lesion. The flap was used in a tube-shaped orientation to replace the urethral section that was removed. An 18-Fr catheter was inserted in the patients for 
the 3 weeks following the surgery. Surgical procedures of the 2 cases were successful and no postoperative complications were observed.

Hematoxylin and eosin-stained sections of the biopsied samples obtained prior to surgery indicated microscopic eosinophilic amorphous deposits in the sub-urothelial lamina propria (Fig. 2A and B). Cells of the tissues, which were stained with Congo red (6) and viewed under a light microscope, were amorphous and characteristically pink-red colored (Fig. 2C and D). The 2 patients were followed up for 24 and 36 months after surgery respectively, and urethroscopic examinations were performed every 6 months in the follow-up periods. Urethroscopic examinations indicated satisfactory outcomes. A total of 6 months after the surgery, urethroscopic examination indicated no evidence of recurrence of the amyloidosis was observed, and the lumens of the artificial urethras were smooth without strictures (Fig. 1B).

\section{Discussion}

A limited number of urethral amyloidosis cases have been reported in the literature, and the cause of this condition remains uncertain. However, the results of previous studies suggest that recurrent chronic urethral inflammation serves an important role in the development of urethral amyloidosis (4). The most common complaints of patients with urethral amyloidosis are hematuria and a bloody urethral discharge, which typically raises suspicion of a malignant urinary tract disease (7). Furthermore, urethrorrhagia during an erection is occasionally noted as amyloidosis may occur in the corpus spongiosum instead of the urothelium (8). Urethral amyloidosis remains difficult to diagnose based on clinical symptoms alone; therefore, endoscopic findings and radiological results are important for confirming a diagnosis. Urethroscopic or surgical biopsies with a frozen section evaluated prior to radical resection are essential for the differential diagnosis and to avoid unnecessary resection (3). In the present case report, the clinical symptoms and urethroscopic findings of the 2 patients suggested a urethral malignancy, and the correct diagnosis was difficult to determine from the available data. However, a lesional biopsy and histological examination aided the diagnosis of urethral amyloidosis and guided treatment.

The current treatment strategies for urethral amyloidosis remain controversial. Certain urologists have indicated that conservative methods are the optimal choice for treatment, as the disease has a benign clinical course and progresses slowly $(1,9)$. For example, urethral dilatation is a conservative approach that has been presented in a number of studies as an effective short-term treatment option $(1,9)$. Furthermore, transurethral resection is an alternative option in cases where dilatation is unsuccessful (1). Unfortunately, recurrence and urethral stricture are common complications following conservative treatment, particularly during long-term follow-up (4). Based on these findings, several studies have suggested more aggressive approaches, such as urethroplasty, to improve treatment outcomes $(5,8)$.

Urethroplasty has been used in a limited number of urethral amyloidosis cases, but these have only included short-term follow-ups $(8,10)$. A follow-up period of $>2$ years has been recommended previously (11). In the 2 patients included in the present case report, urethroplasty was the chosen treatment, and outcomes were followed-up at 24 and 36 months. No signs of urethral stricture or recurrence of amyloidosis on any urethral segments were observed, indicating that urethroplasty is an appropriate approach for the long-term management of urethral amyloidosis.

Pedicled penile skin flaps or buccal mucosa grafts are the most common materials used during urethroplasty due to their higher graft survival rates on the urethra (12). However, it is important to confirm that amyloidosis is localized only in the urethra, as the lesions may also affect the buccal mucosa and penile skin (13). In this situation, urethroplasty should be cautiously chosen as grafted tissue may be affected by amyloidosis postoperatively when the disease is systemic $(8,9)$, and recurrence may occur more frequently (14). In the present report, a pedicled penile skin flap was provided in 1 case, and a pedicled penile skin flap and buccal mucosa graft was applied in the other case. No recurrence of amyloidosis was observed in the grafted tissue at 24 and 36 months following the surgical procedure. These results suggest that urethroplasty is a beneficial long-term treatment for urethral amyloidosis. The 2 patients remain in continuous follow-up. If recurrence occurs in the grafts, the time to recurrence may become an important factor in deciding the appropriate treatment for each case.

In conclusion, the conservative treatment of urethral amyloidosis may lead to recurrence of amyloidosis or a urethral stricture because of the progressive nature of the disease. However, the positive outcomes of the patients included in the present study suggest that urethroplasty is a beneficial treatment for the long-term management of urethral amyloidosis.

\section{Acknowledgements}

The present study was supported by grants from the Scientific Research Fund of the Health Bureau of Zhejiang Province (grant no. 2015KYA072), the Scientific Research Fund of Zhejiang Province Education Department (grant no. Y201636342), and the Scientific Research Fund of Zhejiang Province Traditional Chinese Medical Project of Science and Technology (grant no. 2017ZA081).

\section{References}

1. Sakuma S, Miyazaki T and Hirata H: A case of primary localized amyloidosis of the penile urethra. Int J Urol 3: 163-164, 1996.

2. Sipe JD, Benson MD, Buxbaum JN, Ikeda S, Merlini G, Saraiva MJ, Westermark P and Nomenclature Committee of the International Society of Amyloidosis: Amyloid fibril protein nomenclature: 2012 recommendations from the nomenclature committee of the international society of amyloidosis. Amyloid 19: 167-170, 2012.

3. Zhou F, Lee P, Zhou M, Melamed J and Deng FM: Primary localized amyloidosis of the urinary tract frequently mimics neoplasia: A clinicopathologic analysis of 11 cases. Am J Clin Exp Urol 2: 71-75, 2014.

4. Mangera A, Linton KD, Fernando M, Channer J and Chapple CR: What is the evidence for the management of urethral amyloidosis? A systematic review of the literature. BJU Int 109: 1858-1861, 2012.

5. Tirzaman O, Wahner-Roedler DL, Malek RS, Sebo TJ, Li CY and Kyle RA: Primary localized amyloidosis of the urinary bladder: A case series of 31 patients. Mayo Clin Proc 75: 1264-1268, 2000.

6. Yellapurkar S, Natarajan S, Boaz K, Baliga M, Shetty P, Manaktala N, Prasad M and Ravi M: Tumour-associated tissue eosinophilia in oral squamous cell carcinoma-a boon or a bane? J Clin Diagn Res 10: ZC65-68, 2016. 
7. Biyani CS, Fitzmaurice RJ and Upsdell SM: Localized amyloidosis of the urethra with transitional cell carcinoma of the bladder. BJU Int 83: 722-723, 1999.

8. Cormio L, Sanguedolce F, Pentimone S, Perrone A, Annese P, Turri FP, Bufo P and Carrieri G: Urethral corpus spongiosum amyloidosis presenting with urethrorrhagia during erection. J Sex Med 6: 2915-2917, 2009.

9. Mani S, Flynn SD, Duffy TP and Morgan W: Isolated amyloidosis of the penile urethra and corpus spongiosum: A case report. J Urol 150: 1915-1916, 1993.

10. Madersbacher S and Maier U: Localized amyloidosis of the urethra. Br J Urol 75: 245-246, 1995.

11. Crook TJ, Koslowski M, Dyer JP, Bass P and Birch BR: A case of amyloid of the urethra and review of this rare diagnosis, its natural history and management, with reference to the literature. Scand J Urol Nephrol 36: 481-486, 2002.
12. Kurbatov D, Stojanovic B, Dubskiy S, Lepetukhin A and Djordjevic ML: Buccal mucosa graft urethroplasty in a case of urethral amyloidosis presenting with long anterior urethral stricture. Can Urol Assoc J 9: E830-E833, 2015.

13. Takeda Y, Sekiyama S, Suzuki A and Hirose H: Localized oral amyloidosis: Ultrastructural and immunohistochemical study. J Oral Pathol 16: 278-281, 1987.

14. Kawsar M and Long S: Localized amyloidosis of glans penis. Int J STD AIDS 18: 720-721, 2007. 